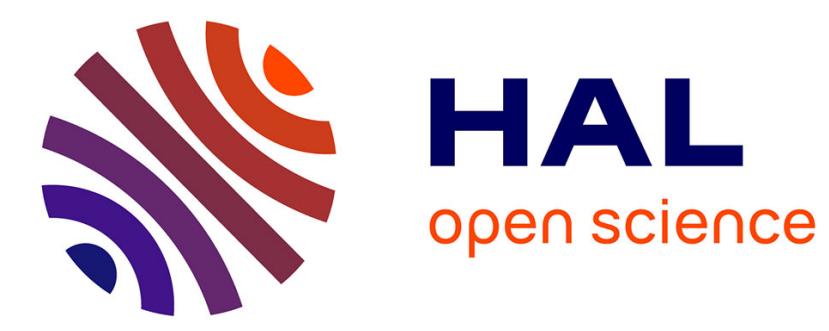

\title{
Calculation of a point source radiation in a flat or non-flat stratified background: an alternative to Sommerfeld integrals
}

\author{
S. Boutami, J. Hazart
}

\section{> To cite this version:}

S. Boutami, J. Hazart. Calculation of a point source radiation in a flat or non-flat stratified background: an alternative to Sommerfeld integrals. European Physical Journal: Applied Physics, 2010, 52 (2), 10.1051/epjap/2010072 . hal-00634372

\section{HAL Id: hal-00634372 \\ https://hal.science/hal-00634372}

Submitted on 21 Oct 2011

HAL is a multi-disciplinary open access archive for the deposit and dissemination of scientific research documents, whether they are published or not. The documents may come from teaching and research institutions in France or abroad, or from public or private research centers.
L'archive ouverte pluridisciplinaire HAL, est destinée au dépôt et à la diffusion de documents scientifiques de niveau recherche, publiés ou non, émanant des établissements d'enseignement et de recherche français ou étrangers, des laboratoires publics ou privés. 


\title{
Calculation of a Point Source radiation in a flat or non-flat stratified background: an alternative to Sommerfeld integrals
}

\author{
S. Boutami and J. Hazart \\ CEA-Léti, Minatec, 17 rue des Martyrs, F38054 Grenoble, France \\ Salim.boutami@cea.fr
}

In this paper, we present an alternative method to Sommerfeld integrals for the calculation of a point source radiation in a multilayered background, based on the method of auxiliary sources, also called fictitious sources method. The method lies upon the decomposition of reflected and transmitted fields on a basis of secondary sources, which amplitudes are determined by applying boundary conditions at each multilayer interface, solving an overdetermined system of equations. We present a generalization of the classical FSM to open stratified domains, with arbitrary number of layers and arbitrary position of the point source in the multilayer. Two and three dimensional formalisms are proposed, and compared to exact Sommerfeld formulation. Computation time reduction factors as high as 100 are obtained for 2D problems. Moreover, this method enables the study of non-flat interfaces, which is not possible with Sommerfeld approach. This method can be used for example in order to determine radiation efficiency of emitting devices, or it can be integrated in discrete source type electromagnetic methods for calculation of scattering by a complex target.

\section{Introduction}

The calculation of the radiation of a point source in a homogeneous medium is straightforward. It is given analytically by Hankel functions of integer order in 2D problems, and half-integer order in 3D problems [1]. However, the radiation calculation of a source in a stratified background becomes more complicated. Sommerfeld first treated the case of a dipole radiation in a half-space background [2], and his method was then extended to multilayer backgrounds [3-7]. The Sommerfeld approach consists in decomposing the source radiation into plane waves by Fourier transform of the Hankel function. The substrate interface is then taken into account by simply applying Fresnel coefficients to each Fourier components (or equivalent reflection and transmission coefficients in case of multilayered background). The field calculation in free space resumes then to an inverse Fourier transform, which leads to the computation of an integral that has generally no analytic solution, and must be computed numerically. This numerical integration is tricky and very time consuming.

It is to be noted that simplifications have been shown to be possible in some specific cases [8].

The first case concerns the radiation of a dipole in a half-space medium very close from the interface [9-10]. In this case, the near-field source radiation can be analytically determined by a quasi-static approximation, neglecting retard effects. Indeed, Sommerfeld integrals resume indeed to Lipschitz integrals, which have an analytic solution [9].

The second case of simplification concerns the far-field radiation of a dipole radiating in a stratified background. In this case, it is sufficient to consider only the plane wave component of the dipole radiation which wavevector is given by the relative positions of source and field calculation point [11]. The third case is the case of a source located above a perfecly conducting ground. In this case, a single image source is sufficient to solve the problem [12]. However, in all cases, only flat interfaces are considered.

The method we propose is, contrary to Sommerfeld method, an indirect method, that consists in expressing the diffracted fields in all layers on a basis of fictitious secondary sources (classical Hankel functions) which amplitudes are not known a priori, and which located in the vicinity of interfaces. The fictitious sources unknown amplitudes are determined by 
applying boundary conditions at all multilayer interfaces, solving a linear system of equations. This method is known as the fictitious sources method (FSM) [13,14] or the method of auxiliary sources (MAS) [15-17]. FSM has been generally used for the calculation of electromagnetic scattering by closed homogeneous targets in free space $[1,13]$. The method we propose is thus a derivation of classical fictitious sources method (FSM) for calculation of source radiation in stratified open domains. We will thus denote it as a stratified backgroundFictitious sources method (SB-FSM), to differentiate it from classical FSM. One can find previous works using FSM for calculation of source radiation above a conductive substrate [18]. In the present paper, our main contribution is the generalization of the principle to an arbitrary number of layers, the source being embedded or not inside the multilayer. Compared to previous works, we also extend the method to 3D problems, and consider non-flat interfaces.

We show that the method, which is a surface method, can be faster than Sommerfeld's one, especially for 2D problems, and is moreover no restricted to flat surfaces.

The applications of SB-FSM are numerous, especially in the field of light emitting devices (Light emitting diodes efficiency, fluorescent molecule radiation, quantum dot radiation...).

After giving the basic principles of the method for the case of a single interface, we will derive it for multiple interfaces, giving examples of $2 \mathrm{D}$ as well as $3 \mathrm{D}$ source radiation calculations.

\section{2D SB-FSM}

\subsection{Single flat interface}

\subsubsection{Theory}

In the problem of a radiation of a source above a substrate, the total field can be formally decomposed into the sum of the primary field of the source and the reflected field in the superstrate where the source is located, and transmitted field in substrate. The Sommerfeld method consists in a direct evaluation of the reflected and transmitted fields, whereas SBFSM consists in an indirect evaluation.

In SB-FSM, the reflected field is expanded on a set of secondary fictitious sources located in the substrate and radiating in the superstrate, whereas the transmitted field is expanded on a set of secondary sources located in the superstrate and radiating in the substrate (see Figure $1)$.

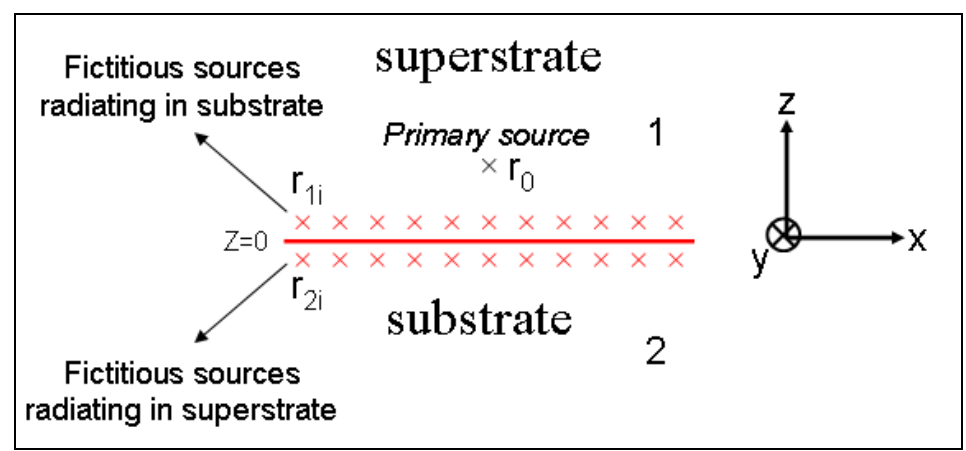

Fig. 1: Location of sets of fictitious secondary sources in SB-FSM for a single interface background. 
The fictitious sources are thus characterized by classical free-space $0^{\text {th }}$-order Hankel functions, radiating in a homogeneous medium (as the primary source). The problem of singularity of the fictitious sources is avoided since the fictitious sources are not located in the medium where they radiate.

SB-FSM is a monochromatic method (frequency $\omega$ ). We suppose a $e^{-i \omega t}$ temporal dependence of field.

The Hankel function $\mathrm{H}_{0}{ }^{(1)}$ is the fundamental solution of the Helmholtz equation with a pointsource term, and satisfying radiation condition in $2 \mathrm{D}$ :

$\Delta H_{0}{ }^{(1)}+k^{2} H_{0}{ }^{(1)}=4 i \delta\left(r-r_{0}\right)$

where $r_{0}$ is the spatial origin of the Hankel function

In $2 \mathrm{D}$ problems, Helmholtz equation is satisfied by the longitudinal electric field component Ey, whereas in TM it is satisfied by Hy.

Let us focus on TE problems. Hankel functions express thus the longitudinal Ey scalar field radiated by the source. The SB-FSM formulation can be written:

$$
\begin{aligned}
& E_{y}{ }^{1}(\vec{r})=H_{0}{ }^{(1)}\left(k_{1}\left\|\vec{r}-\overrightarrow{r_{0}}\right\|\right)+\sum_{i=0}^{N s} c_{2 i} \cdot H_{0}{ }^{(1)}\left(k_{1}\left\|\vec{r}-\overrightarrow{r_{2 i}}\right\|\right) \\
& E_{y}{ }^{2}(\vec{r})=\sum_{i=0}^{N s} c_{1 i} H_{0}{ }^{(1)}\left(k_{2}\left\|\vec{r}-\overrightarrow{r_{1 i}}\right\|\right)
\end{aligned}
$$

where:

$\overrightarrow{r_{1 i}}$ and $\overrightarrow{r_{2 i}}$ denote respectively the location of substrate and superstrate secondary fictitious sources

$k_{1}=n_{1} \frac{2 \pi}{\lambda}$ and $k_{2}=n_{2} \frac{2 \pi}{\lambda}$ denote respectively superstrate and substrate wavevectors, $\lambda$ being the wavelength

Ns is the number of secondary fictitous sources in each medium

$c_{1 i}$ (resp. $c_{2 i}$ ) denote the unknown complex amplitudes of superstrate (resp. susbtrate) fictitious sources

The magnetic field $\vec{H}$ can then be determined from Maxwell equation in time-harmonic regime:

$$
\nabla \times \vec{E}=i \omega \mu \overrightarrow{\mathrm{H}}
$$

which leads, when solved in polar coordinates, to:

$$
\begin{aligned}
& \mathrm{H}_{\varphi}{ }^{1}(\vec{r})=-i \sqrt{\frac{\varepsilon_{0}}{\mu_{0}}} \sqrt{\frac{\varepsilon_{1}}{\mu_{1}}}\left(H_{1}{ }^{(1)}\left(k_{1}\left\|\vec{r}-\overrightarrow{r_{0}}\right\|\right)+\sum_{i=0}^{N s} c_{2 i} H_{1}^{(1)}\left(k_{1}\left\|\vec{r}-\overrightarrow{r_{2 i}}\right\|\right)\right) \\
& \mathrm{H}_{\varphi}{ }^{2}(\vec{r})=-i \sqrt{\frac{\varepsilon_{0}}{\mu_{0}}} \sqrt{\frac{\varepsilon_{2}}{\mu_{2}}}\left(\sum_{i=0}^{N s} c_{1 i} H_{1}^{\left({ }^{(1)}\right.}\left(k_{2}\left\|\vec{r}-\overrightarrow{r_{1 i}}\right\|\right)\right)
\end{aligned}
$$

The determination of the sources unknown amplitudes $c_{1 i}$ and $c_{2 i}$ is done numerically. We solve on $\mathrm{Nm}$ discrete points of the interface, namely matching points [1], the tangential and normal field continuity conditions. Maxwell equations impose $\mathrm{H}$ and $\mathrm{E}$ tangential components, and D and B normal components to be continuous through the interface. Note that $\mathrm{H}$ needs to be normalized by the vacuum impedance in order to be of the same order of magnitude as $E$ in the linear system of equations.

It leads in $2 \mathrm{D}$ to 3 set of $\mathrm{Nm}$ scalar equations: 
$E_{y}{ }^{1}\left(r_{m i}\right)=E_{y}{ }^{2}\left(r_{m i}\right)$ for $\mathrm{i}=1 . . \mathrm{Nm}$

$\overrightarrow{n_{i}} \times \overrightarrow{\mathrm{H}^{1}\left(\overrightarrow{r_{m i}}\right)}=\overrightarrow{n_{i}} \times \overrightarrow{\mathrm{H}^{2}\left(\overrightarrow{r_{m i}}\right)} \quad$ for $\mathrm{i}=1 . . \mathrm{Nm}$

$\overrightarrow{n_{i}} \cdot\left(\mu_{1} \overrightarrow{\mathrm{H}^{1}\left(\overrightarrow{r_{m i}}\right)}\right)=\overrightarrow{n_{i}} \cdot\left(\mu_{2} \overrightarrow{\mathrm{H}^{2}\left(\overrightarrow{r_{m i}}\right)}\right) \quad$ for $\mathrm{i}=1 . . \mathrm{Nm}$

Where $r_{\text {mi }}$ denotes the location of the $i^{\text {th }}$ matching point.

$\mathrm{Nm}$ has to be chosen sufficiently high in order that the meshed interface is close to the real one, i.e. in order that the solution obtained satisfies boundary conditions on the whole real interface (which means also between matching points). It is known that the spatial oscillation frequency of field expansions (2) and (3) increases with the number of fictitious sources Ns. Therefore, we overdetermine the linear system of equation (the Matrix associated with the linear system of equations is rectangular). Generally, taking $\mathrm{Nm}=2 * \mathrm{Ns}$ leads to three times as many equations as unknowns, and guarantees a smooth behaviour of the solution between matching points. The overdetermined system is solved in the least square sense. The residual error obtained, which represents the boundary continuity error, gives a good insight on the solution accuracy.

The exact solution corresponds to an infinite number of secondary sources (except for the radiation of a source above a perfectly conducting substrate, which only requires one single secondary source). So, generally, the expansion is not a complete basis, and the truncation of the number of sources induces an error, but this error decreases exponentially with the number of secondary sources. The values of permittivities of the different media affect the convergence rate. Indeed, high permitivity media generally require higher densities of secondary sources.

In addition to the truncation of the number of secondary sources, another origin of error is the lateral spatial truncation of the set of secondary sources and interface meshing. Contrary to Sommerfeld method which is based on a plane wave decomposition that considers infinite interfaces, the SB-FSM method requires the interfaces to be laterally truncated. We will show that it limits the spatial area of validity of the solution obtained.

\subsubsection{Example}

We consider the case of a point-source located in a superstrate (medium1, $\mu=1$ and $\varepsilon=2.25$ ) at a distance of $\lambda / 2$ from a substrate of air (medium $2, \mu=1$ and $\varepsilon=1$ ), the interface being flat.

Let us consider the exact computation of such a problem, by invoking Sommerfeld integrals. For $2 \mathrm{D}$, problems, it can be shown that the plane wave expansion of the $0^{\text {th }}$-order Hankel function in a homogenous medium is [19-23]:

$$
H_{0}^{(1)}\left(k\left\|\vec{r}-\overrightarrow{r_{0}}\right\|\right)=\frac{1}{\pi} \int_{-\infty}^{+\infty} \frac{e^{i\left(\sqrt{k^{2}-k_{x}{ }^{2}}\left|z-z_{0}\right|+k_{x}\left(x-x_{0}\right)\right)}}{\sqrt{k^{2}-k_{x}^{2}}} d k_{x}
$$

Where $k_{x}$ and $\sqrt{k^{2}-k_{x}^{2}}$ represents respectively the horizontal and vertical wavevector components.

$\overrightarrow{r_{0}}=\left(x_{0}, z_{0}\right)$ is the location of the source. 
For TE problems, we remind that it is Ey that is expressed by (10). In presence of the interface, the field in superstrate and substrate can be obtained applying TE reflection and transmission Fresnel coefficients to each Fourier component:

$$
\begin{aligned}
& E_{y}{ }^{1}(\vec{r})=\frac{1}{\pi} \int_{-\infty}^{+\infty}\left[\frac{e^{i\left(\sqrt{k_{1}^{2}-k_{x}{ }^{2}}\left|z-z_{0}\right|+k_{x}\left(x-x_{0}\right)\right)}}{\sqrt{k_{1}{ }^{2}-k_{x}^{2}}}+r_{s}\left(k_{x}\right) * \frac{e^{i\left(\sqrt{k_{1}{ }^{2}-k_{x}}\left(|z|+\left|z_{0}\right|\right)+k_{x}\left(x-x_{0}\right)\right)}}{\sqrt{k_{1}^{2}-k_{x}^{2}}}\right] d k_{x} \\
& E_{y}{ }^{2}(\vec{r})=\frac{1}{\pi} \int_{-\infty}^{+\infty} t_{s}\left(k_{x}\right) * \frac{e^{i\left(\sqrt{k_{1}{ }^{2}-k_{x}{ }^{2}}|z|+\sqrt{k_{2}{ }^{2}-k_{x}{ }^{2}}\left|z_{0}\right|+k_{x}\left(x-x_{0}\right)\right)}}{\sqrt{k_{1}^{2}-k_{x}^{2}}} d k_{x}
\end{aligned}
$$

Where :

$$
\begin{gathered}
r_{s}\left(k_{x}\right)=\frac{\sqrt{k_{1}^{2}-k_{x}^{2}}-\sqrt{k_{2}^{2}-k_{x}^{2}}}{\sqrt{k_{1}^{2}-k_{x}^{2}}+\sqrt{k_{2}^{2}-k_{x}^{2}}} \\
t_{s}\left(k_{x}\right)=\frac{2 \sqrt{k_{1}^{2}-k_{x}^{2}}}{\sqrt{k_{1}^{2}-k_{x}^{2}}+\sqrt{k_{2}^{2}-k_{x}^{2}}}
\end{gathered}
$$

are the TE reflection and transmission Fresnel coefficients for horizontal wavevector component kx.

The magnetic field components can be obtained by applying equation (4) in Cartesian coordinates:

$$
\begin{aligned}
& H_{x}{ }^{1}(\vec{r})=-\sqrt{\frac{\varepsilon_{0}}{\mu_{0}}} \frac{1}{\mu_{1} k_{0}} \frac{1}{\pi} \int_{-\infty}^{+\infty}\left[\operatorname{sign}\left(z_{0}-z\right) * e^{i\left(\sqrt{k_{1}^{2}-k_{x}^{2}}\left|z-z_{0}\right|+k_{x}\left(x-x_{0}\right)\right)}+r_{s}\left(k_{x}\right) * e^{i\left(\sqrt{k_{1}{ }^{2}-k_{x}{ }^{2}}\left(z|+| z_{0} \mid\right)+k_{x}\left(x-x_{0}\right)\right)}\right] d k_{x} \\
& H_{x}^{2}(\vec{r})=-\sqrt{\frac{\varepsilon_{0}}{\mu_{0}}} \frac{1}{\mu_{2} k_{0}} \frac{1}{\pi} \int_{-\infty}^{+\infty}\left[t_{s}\left(k_{x}\right) * e^{i\left(\sqrt{k_{1}^{2}-k_{x}}\left|z_{0}\right|+\sqrt{k_{2}{ }^{2}-k_{x}^{2}}|z|+k_{x}\left(x-x_{0}\right)\right)}\right] d k_{x} \\
& H_{z}{ }^{1}(\vec{r})=\sqrt{\frac{\varepsilon_{0}}{\mu_{0}}} \frac{1}{\mu_{1} k_{0}} \frac{1}{\pi} \int_{-\infty}^{+\infty}\left[\frac{e^{i\left(\sqrt{k_{1}{ }^{2}-k_{x}}\left|z-z_{0}\right|+k_{x}\left(x-x_{0}\right)\right)}}{\sqrt{k_{1}{ }^{2}-k_{x}^{2}}}+r_{s}\left(k_{x}\right) * \frac{e^{i\left(\sqrt{k_{1}{ }^{2}-k_{x}}\left(|z|+\left|z_{0}\right|\right)+k_{x}\left(x-x_{0}\right)\right)}}{\sqrt{k_{1}{ }^{2}-k_{x}{ }^{2}}}\right] k_{x} d k_{x} \\
& H_{z}^{2}(\vec{r})=\sqrt{\frac{\varepsilon_{0}}{\mu_{0}}} \frac{1}{\mu_{2} k_{0}} \frac{1}{\pi} \int_{-\infty}^{+\infty} t_{s}\left(k_{x}\right) * \frac{e^{i\left(\sqrt{k_{1}{ }^{2}-k_{x}}\left|z_{0}\right|+\sqrt{k_{2}{ }^{2}-k_{x}}|z|+k_{x}\left(x-x_{0}\right)\right)}}{\sqrt{k_{1}^{2}-k_{x}^{2}}} k_{x} d k_{x}
\end{aligned}
$$

Figure 2 shows the Sommerfeld computation of the point source radiation in presence of the substrate, compared with that it would have been in a homogeneous medium (considered as the superstrate medium). All dimensions in figures are normalized by $\lambda$. 


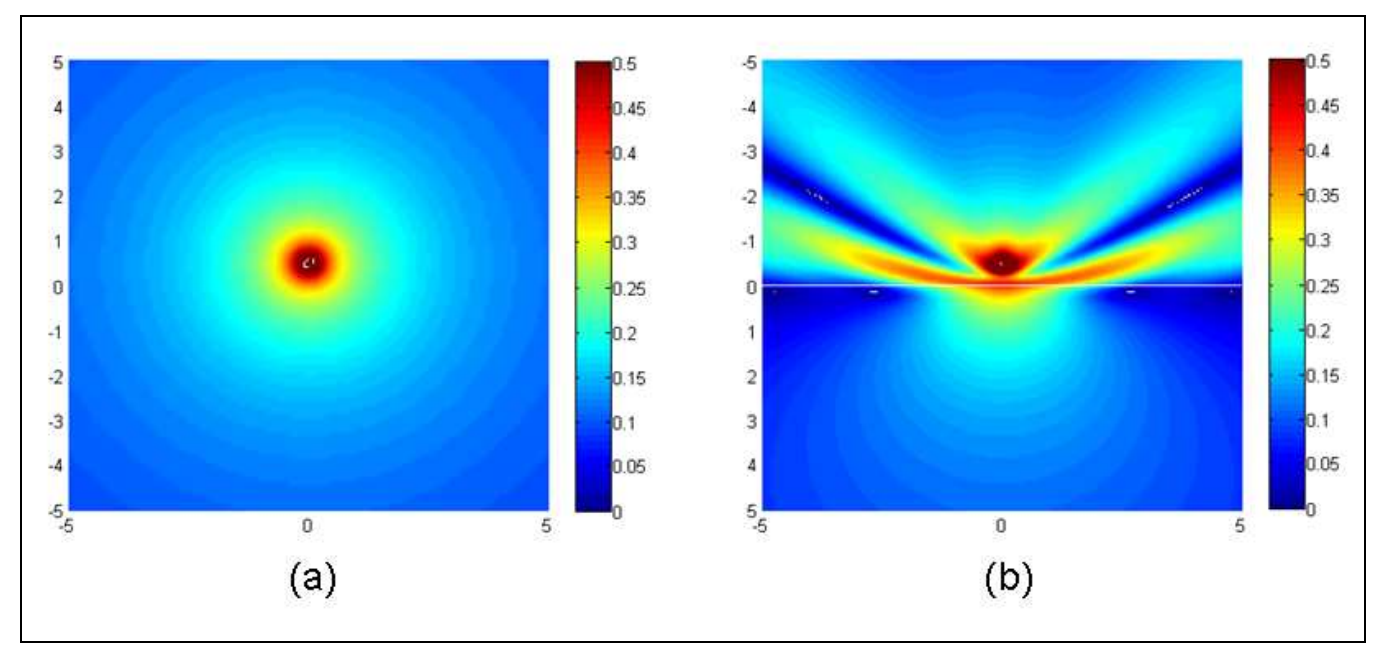

Fig. 2: radiation of a $2 \mathrm{D}$ source: (a) in a homogeneous background ( $\mu=1$ and $\varepsilon=2.25$ );(b) in presence of a air substrate ( $\mu=1$ and $\varepsilon=2.25$ ) (Sommerfeld integrals calculation)

Note that Sommerfeld integrals have been computed with a relative accuracy of 10-6.

Let us now solve the problem by the SB-FSM. Sommerfeld formulations (11) and (12) are valid in whole space. In SB-FSM, the interface has to be laterally truncated. We will now examine the influence of this truncation on the accuracy of the solution obtained.

The boundary length taken into account is $5 \lambda$. In the case of flat or smooth interface, it can be shown that best convergences are obtained for fictitious source positioned along a line (surface in 3D) conformal to the interface [24]. It is to be mentioned that the distance of the fictitious sources to the interface is a degree of freedom that can affect the convergence rate [14], i.e. the continuity error for a given number of secondary sources Ns. In the present example, we chose a distance of $\lambda$ between the sources and the interface.

In Figure 3 is plotted the longitudinal electric field Ey for different number of fictitious sources: $\mathrm{Ns}=10, \mathrm{Ns}=30$, and $\mathrm{Ns}=50$. Corresponding average boundary continuity errors are given in each case. The boundary continuity errors are normalized with respect to the primary field intensity taken at each matching point. We can see that the convergence is obtained as soon as Ns is superior to a threshold that is around 50. Increasing Ns above 50 would lead to an exponential decreasing of the error. 


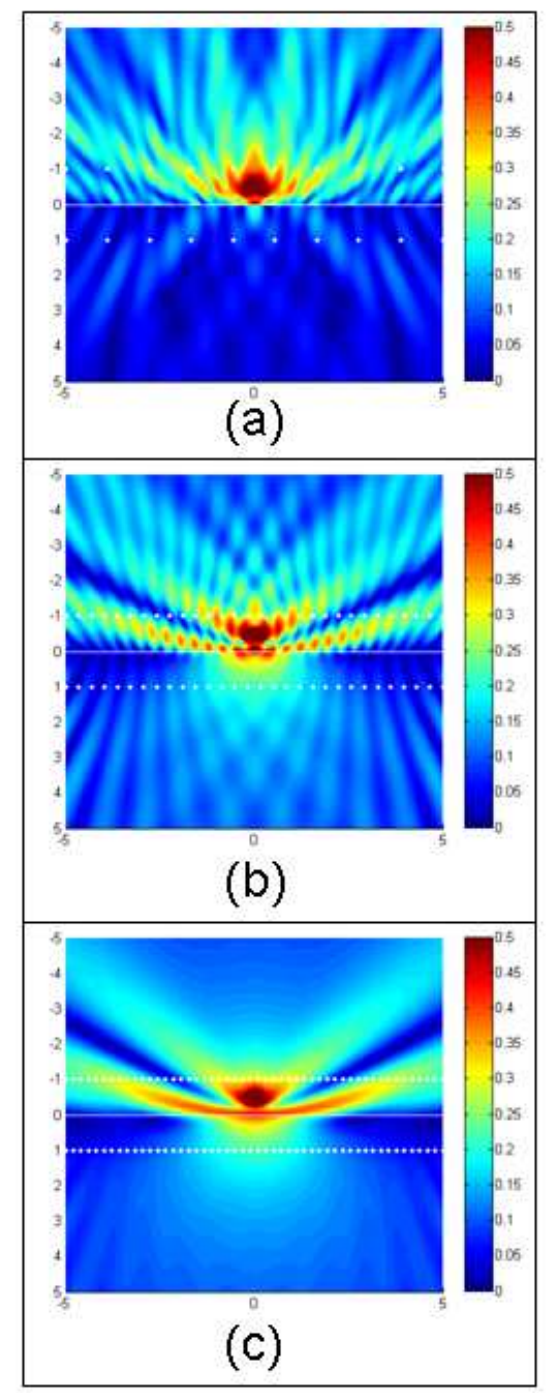

Fig. 3: Ey field calculated by SB-FSM for the case of Fig. $2 b$ for (a) Ns=10; (b) Ns=30; (c) $\mathrm{Ns}=50$. Corresponding average interface continuity error normalized by primary source intensity are respectively (a) $75 \%$; (b) $35 \%$; (c) $10^{-3}$;

For Ns $=50$, the average boundary continuity error is about $10^{-3}$. The relative error in the two dimensional space between the Sommerfeld field computation (Fig. 2b) and the SB-FSM formulation (Fig.3c) is shown on figure 4. It is smaller than $1 \%$ in a region of space that is determined by the interface lateral truncation. This domain of validity is the area of greatest influence of the secondary sources that we have taken into account. When getting further from this area, the omission of lateral sources has a detrimental impact on the accuracy of the solution. One should note that as the field radiated by the point source decreases with the distance, the absolute error remains low.

In conclusion, better accuracies can be obtained by increasing both Ns and the interface length, depending on the area in which field should be known. It is to be mentioned than in figure 4, the area of validity of the SB-FSM computation is sufficiently large to give access to the far-field properties of the source radiation. 


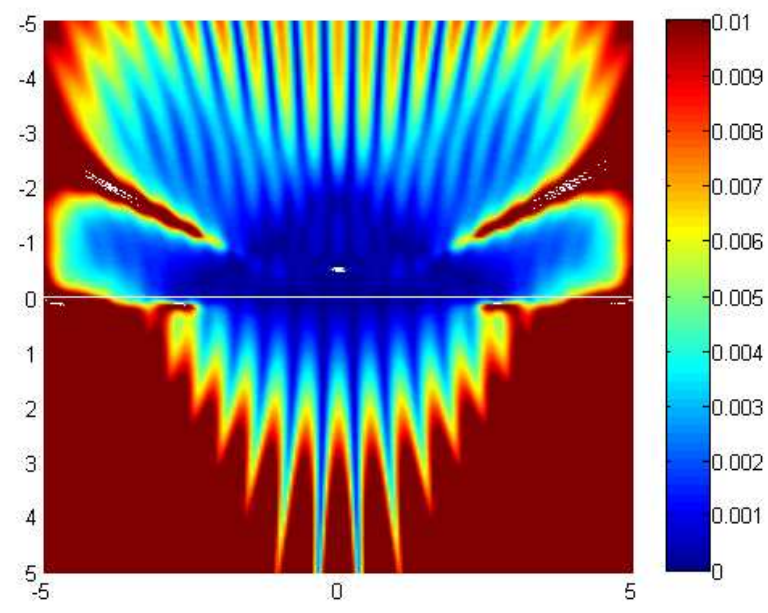

Fig. 4: relative error between Sommerfeld field computation (fig. 2b) and SB-FSM computation (fig. 3c)

\subsection{Flat multilayers}

\subsubsection{Theory}

In the case of multilayered background, the field in each inner layer results from multiple reflections and transmissions. However, the approach presented in section 2.1 for a single interface can be adapted. The procedure is shown in Figure 5.

We keep the secondary sources related to diffracted fields in superstrate and substrate, and we associate to each inner layers two new set of external secondary sources, one located above the layer and expressing field reflected by the partial multilayer composed of all upper layers, and one located below the layer, and expressing field reflected by the partial multilayer composed of all lower layers. 


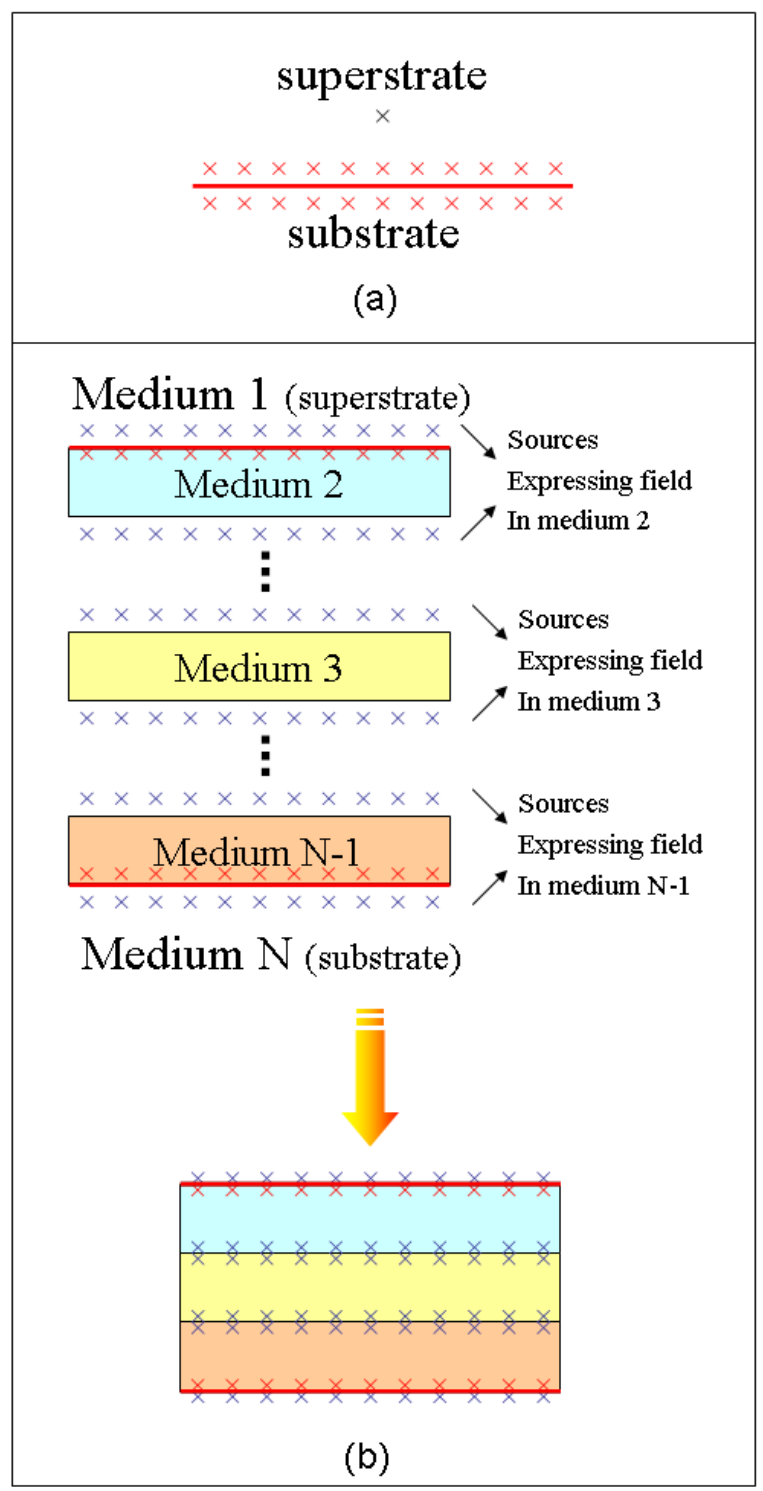

Figure 5: Setting of secondary sources (a) for a single interface stratified background ;(b) for a multilayered stratified background

For a N-media system, we have $2(\mathrm{~N}-1)$ sets of secondary sources. We then solve the boundary continuity conditions at all interfaces of the multilayer. Due to the construction of sets of secondary sources, each interface boundary conditions involve only a few sets of sources; 3 sets for the boundaries of substrate or superstrate, and 4 sets of sources for other interfaces.

Indeed, the fields in the semi-infinite substrate and superstrate are each expressed by a single set of source, as can be seen in figure 5.b (red set of source). On the other hand, the field in each layer is expressed by two set of sources. Therefore, the boundary equations applied on substrate or superstrate interfaces involve three set of sources, whereas boundary equations applied on interfaces between layers involve four set of sources.

The problem finally resumes to the resolution of a system of coupled equations. The system is made here also overdetermined, and solved in the least-square sense.

\subsubsection{Example}


We take the example of a point source located in a superstrate (medium $1, \mu=1$ and $\varepsilon=2.25$ ), at a distance $\lambda / 2$ above a layer of thickness $\lambda$ (medium $2, \mu=1$ and $\varepsilon=4$ ), and a substrate (medium 3, $\mu=1$ and $\varepsilon=6.25$ ) (see Fig.6).

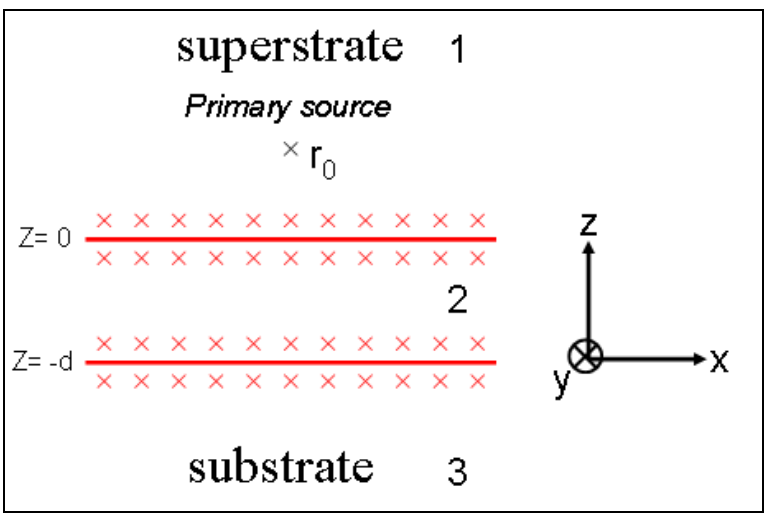

Figure 6: Setting of secondary sources for a primary source radiating in stratified background composed of three media (the primary source is located in the superstrate)

For such a system, the exact calculation (Sommerfeld integrals) is quite similar to that of precedent section, except that we do not take into account Fresnel interface coefficients, but equivalent reflection and transmission multilayers coefficients. These multilayer plane wave coefficients can be calculated by a transfert matrix approach [25], and depend on all reflection Fresnel coefficients and layer thicknesses of the multilayer.

The Ey field is then expressed in the three media by:

$$
\begin{aligned}
& E_{y}(\vec{r})=\frac{1}{\pi} \int_{-\infty}^{+\infty}\left[\frac{e^{i\left(\sqrt{k_{1}^{2}-k_{x}^{2}}\left|z-z_{0}\right|+k_{x}\left(x-x_{0}\right)\right)}}{\sqrt{k_{1}^{2}-k_{x}^{2}}}+A_{1}\left(k_{x}\right) * \frac{e^{i\left(\sqrt{k_{1}^{2}-k_{x}^{2}}\left(|z|+\left|z_{0}\right|\right)+k_{x}\left(x-x_{0}\right)\right)}}{\sqrt{k_{1}^{2}-k_{x}^{2}}}\right] d k_{x} \\
& E_{y}{ }^{2}(\vec{r})=\frac{1}{\pi} \int_{-\infty}^{+\infty}\left[A_{2}\left(k_{x}\right) * \frac{e^{i\left(\sqrt{k_{1}{ }^{2}-k_{x}{ }^{2}}\left|z_{0}\right|-\sqrt{k_{2}{ }^{2}-k_{x}{ }^{2}}|z|+k_{x}\left(x-x_{0}\right)\right)}}{\sqrt{k_{1}{ }^{2}-k_{x}^{2}}}+B_{2}\left(k_{x}\right) * \frac{e^{i\left(\sqrt{k_{1}{ }^{2}-k_{x}{ }^{2}}\left|z_{0}\right|+\sqrt{k_{2}{ }^{2}-k_{x}}|z|+k_{x}\left(x-x_{0}\right)\right)}}{\sqrt{k_{1}{ }^{2}-k_{x}^{2}}}\right] d k_{x} \\
& E_{y}^{3}(\vec{r})=\frac{1}{\pi} \int_{-\infty}^{+\infty} B_{3}\left(k_{x}\right) \frac{e^{i\left(\sqrt{k_{1}{ }^{2}-k_{x}{ }^{2}}\left|z_{0}\right|+\sqrt{k_{3}{ }^{2}-k_{x}{ }^{2}}|z+d|+k_{x}\left(x-x_{0}\right)\right)}}{\sqrt{k_{1}{ }^{2}-k_{x}^{2}}} d k_{x}
\end{aligned}
$$

Where:

$A_{i}$ and $B_{i}$ denote amplitudes of upgoing and downgoing waves for kx horizontal wavevector component in medium $\mathrm{i}$ (TE polarization).

$\mathrm{d}$ denotes the thickness of medium 2

We remind that $\mathrm{kx}$ is continuous through all interfaces.

The Ey field obtained from exact Sommerfeld calculation is shown in figure 7a (Sommerfeld integrals computation with a relative accuracy of $10^{-6}$ ).

For SB-FSM computation, the interface taken into account has a length of $5 \lambda$. The Ey field is plotted in figure $7 \mathrm{~b}$, with $\mathrm{Ns}=100$ for all set of secondary sources. The corresponding average boundary error is around $10^{-8}$. 


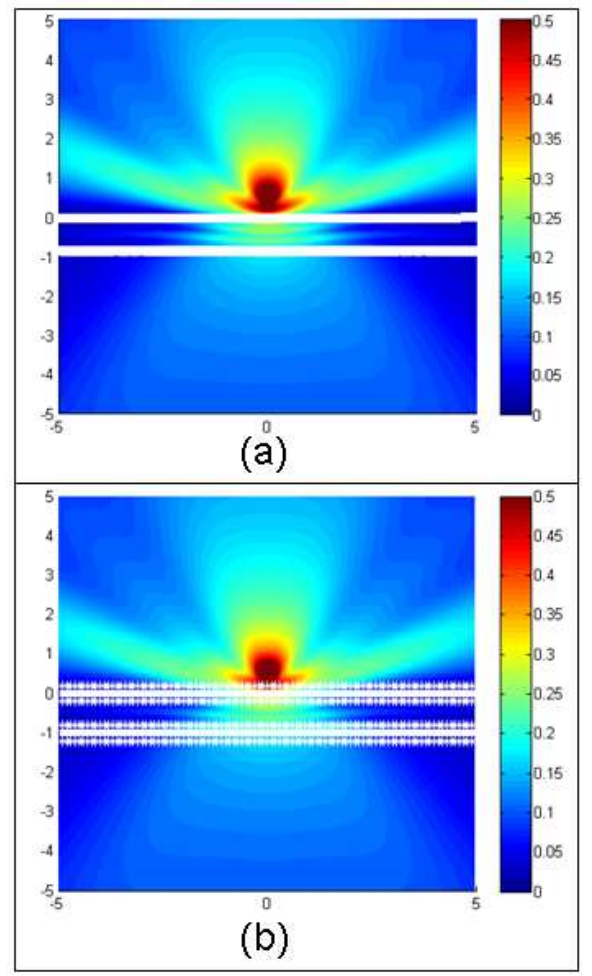

Figure 7: Calculation of Ey field radiated by a source located in medium 1 ( $\mu=1$ and $\varepsilon=2.25$ ), at a distance $\lambda / 2$ above a layer of thickness $\lambda$ (medium $2, \mu=1$ and $\varepsilon=4$ ), and a substrate (medium 3, $\mu=1$ and $\varepsilon=6.25$ ). (a) Sommerfeld calculations; (b) SB-FSM calculations

The spatial relative comparison of Sommerfeld and SB-FSM formulations (Fig. 8) shows that the area of validity of the fictitious sources expansion is again given by the boundary lateral truncation. The relative error is around $10^{-6}$, which means that, for Ns=100, SB-FSM calculation is in the same order of accuracy as Sommerfeld calculation.

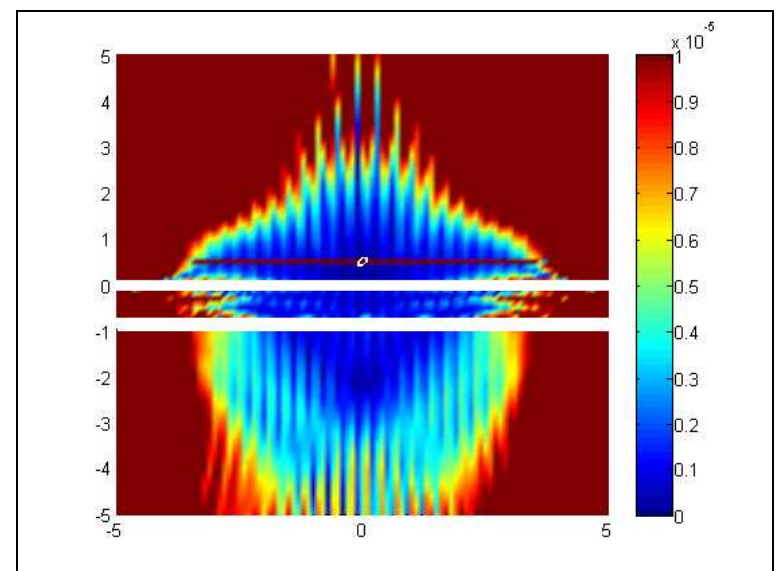

Figure 8: Spatial relative comparison between SB-FSM calculations (Fig. 7b) and Sommerfeld calculations (Fig. 7a) 
For this example, the computation time reduction factor was about 100 compared with Sommerfeld approach. The computation time factor depends on the spatial resolution of the field mapping. In the present case, we have 100x100 points. For higher resolutions, the computation time reduction factor is even better, since the field is known analytically in SBFSM, whereas it has to be evaluated numerically by an integral at each point of the working space in Sommerfeld approach.

It is to be mentioned that it is also possible to perform the calculation for a point-source placed inside a layer. The approach remains strictly the same (same sets of secondary sources), except that this time the primary field holds in the layer, and no more in the superstrate.

Figure9 shows the simulation of such a configuration, the source being located in the middle of the layer.

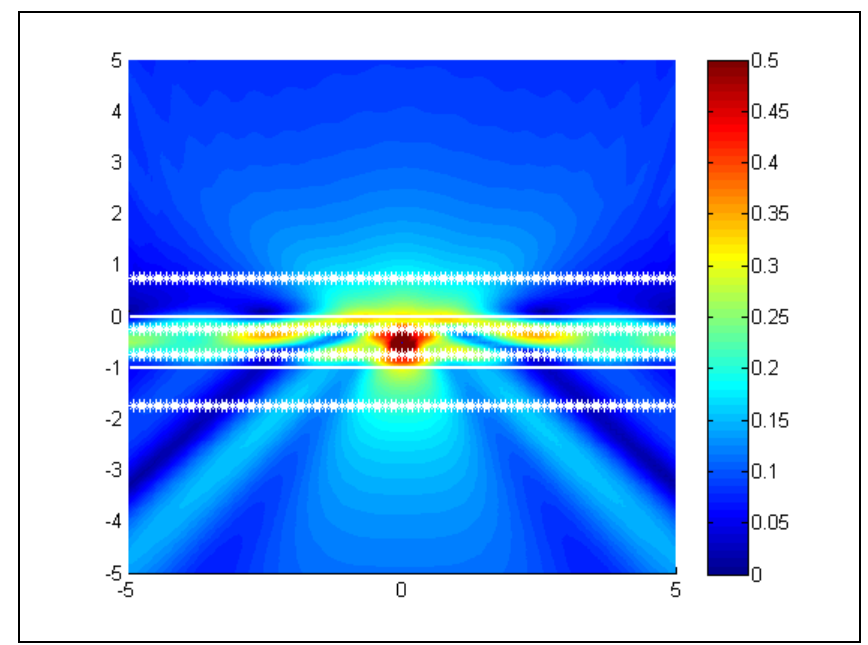

Figure 9: SB-FSM simulation of the structure of figure 6, the primary source being this time located in the middle of the layer. Medium 1 ( $\mu=1$ and $\varepsilon=2.25)$, Medium 2 ( $\mu=1$ and $\varepsilon=4$ ), and Medium 3 ( $\mu=1$ and $\varepsilon=6.25)$.

\section{3 non-flat multilayers}

One specific advantage of the SB-FSM is that it can handle non flat interfaces. One just has to specify the proper normal directions at each matching points of the interfaces.

We have considered the structure of figure 6 , with a sinusoidal corrugation of interfaces characterized by a period $\mathrm{p}=\lambda$, and a variable height $\mathrm{h}$ (from 0 to $0.1 \lambda$ ) (see Fig. 10).

Smooth profiles can be handled with a location of secondary sources conformal to the interfaces. For profiles with faster spatial variations, one should set sources closer to the interface where geometrical singularities occur, as in classical FSM [24]. 


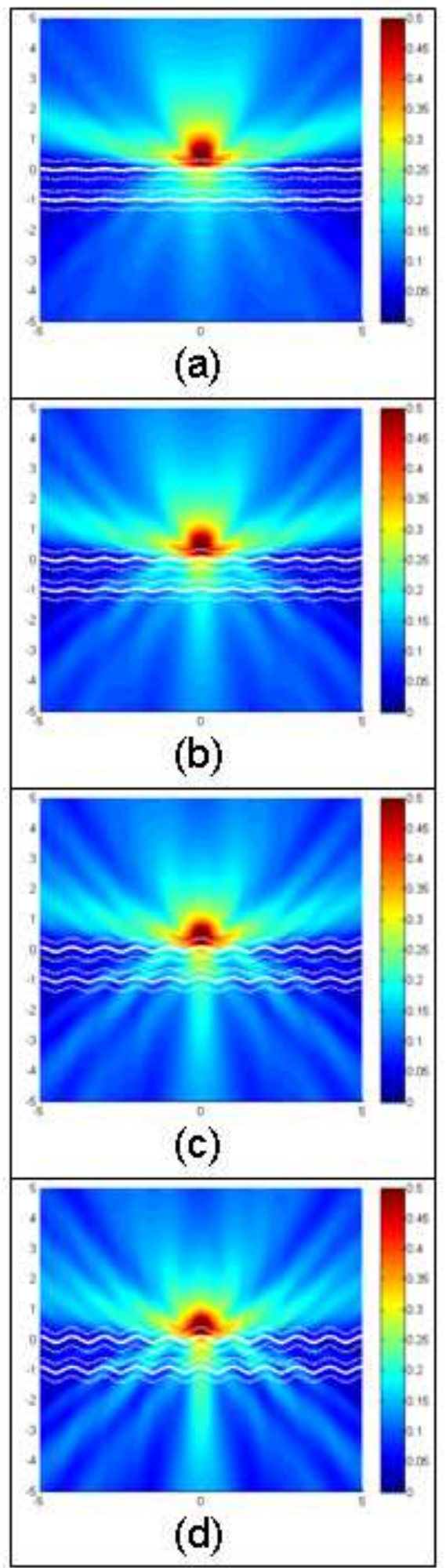

Figure 10: SB-FSM simulation of structure of Fig. 6, but with a sinusoidal corrugation of period $\mathrm{p}=\lambda$ and height: (a) $0.025 \lambda$; (b) $0.05 \lambda$; (c) $0.075 \lambda$; (d) $0.1 \lambda$

Using $\mathrm{Ns}=100$ for each set of secondary sources, the boundary continuity error remains lower than $1 \%$ in each case of corrugation height. The different field patterns are shown in figure 10 , and have to be compared with the flat case (Figure 7). We can thus evaluate the modification of the field due to the corrugation of the film.

Note that the interface profile should not be necessarily periodic with SB-FSM. 


\section{Extension to 3D SB-FSM}

\subsection{Theory}

The 2D point-source we have dealt with in the previous section corresponds to the field radiated by an infinite line source.

In $3 \mathrm{D}$, the elementary point-source is the dipole. It is the model associated with the radiation of small emitter like quantum dots or fluorescent molecules for example.

A dipole is characterized by its wavelenth of emission, but also its orientation. In the present work, we will consider electric dipoles with length negligible compared to wavelength.

In spherical coordinates $(r, \theta, \phi)$, the fundamental solution of Helmholtz equation (1) with a point-source term is the function with a spherical Hankel function radial dependence $h_{1}(\mathrm{kr})$, a $\cos (\theta)$ theta-dependence, and a uniform $\phi$-dependence, where $\theta$ denotes the angle made by $\vec{r}$ and the dipole orientation. When looking for a field component satisfying Helmholtz equation, one can works with the scalar or vector potential. But one can remark that $\mathrm{rEr}$ also satisfies Helmholtz equation [1], which leads to expressions:

$E_{r}(\vec{r})=\frac{1}{r} h_{1}\left(k\left\|\vec{r}-\overrightarrow{r_{0}}\right\|\right) \cos (\theta)$

$E_{\theta}(\vec{r})=-\frac{1}{2 r^{2}} \frac{\partial\left(r h_{1}(k r)\right)}{\partial r} \sin (\theta)$

$H_{\psi}(\vec{r})=-\frac{i \pi}{\lambda} \sqrt{\frac{\varepsilon_{0}}{\mu_{0}}} \mathcal{E} \cdot h_{1}(k r) \sin (\theta)$

Spherical Hankel function $\mathrm{h}_{1}(\mathrm{kr})$ can be expressed in terms of simple trigonometric function of $\mathrm{kr}$ :

$h_{1}(k r)=\frac{\sin (k r)}{(k r)^{2}}-\frac{\cos (k r)}{k r}-i \cdot\left(\frac{\cos (k r)}{(k r)^{2}}+\frac{\sin (k r)}{k r}\right)$

One should note that there are also expressions of dipole radiation in cartesian coordinates, given by the so-called homogeneous Green's tensor [3,9].

In 3D, we find that secondary sources cannot be single dipole, but two or three orthogonal dipoles. Actually, two orthogonal dipoles both tangential to the interface give good results as elementary secondary source $[17,26]$.

Another difference compared with 2D is that the boundary continuity conditions are no more scalar, but vectorial, and lead therefore to $8 \mathrm{Nm}$ interface continuity equations.

\subsection{Example}

We consider the example of a Z-oriented dipole located in a medium 1 ( $\mu=1$ and $\varepsilon=2.25$ ), at a distance $\lambda / 60$ above a flat absorbing layer of thickness $\lambda / 60$ (medium $2, \mu=1$ and $\varepsilon=3+4 i$ ), and a substrate (medium $3, \mu=1$ and $\varepsilon=6.25$ ).

The exact calculation of this problem consists in evaluating the 3D Sommerfeld integrals. We refer the reader to [3] for details about 3D Sommerfeld integrals. We give the form of the Ez field in the superstrate:

$$
E_{z}{ }^{1}(\vec{r})=\left(E_{z}{ }^{1}(\vec{r})\right)_{\text {primary }}+\frac{1}{k_{1}{ }^{2}} \int_{0}^{\infty} A_{1}\left(k_{\rho}\right) \cdot \frac{k_{\rho}{ }^{3}}{\sqrt{k_{1}{ }^{2}-k_{\rho}^{2}}} J_{0}\left(k_{\rho} \rho\right) e^{i \sqrt{k_{1}{ }^{2}-k_{\rho}{ }^{2}}\left(z_{0}+z\right)} d k_{\rho}
$$


Where $k_{\rho}$ denotes the in-plane wavevector Fourier component, $\mathrm{J}_{0}$ is the $0^{\text {th }}$ order Bessel function, and $A_{1}\left(k_{\rho}\right)$ is the $\mathrm{p}$ polarization multilayer reflection coefficient for $k_{\rho}$ Fourier component at the upper interface.

Similar expressions are obtained for other media, with corresponding reflection or transmission multilayer coefficients and removing primary field contribution.

Note that the integral is one-dimensional, as in 2D problems.

As in 2D, the computation of the Sommerfeld integral must be treated carefully in presence of guided modes, which constitute poles for which the denominator of the integrand vanishes. There are also branch cuts related to radiation modes. For computation, one should deform the integration path in the complex plane in order to avoid singularities [3].

Note that a $\mathrm{Z}$ _oriented dipole only radiates p plane wave components. For a horizontal dipole, s-polarized components should be also taken into account, with their corresponding reflection and transmission multilayer coefficients.

The Sommerfeld calculation of the Ez field in the $\mathrm{XZ}$ plane is shown on figure 11, with a relative accuracy of $10^{-6}$.

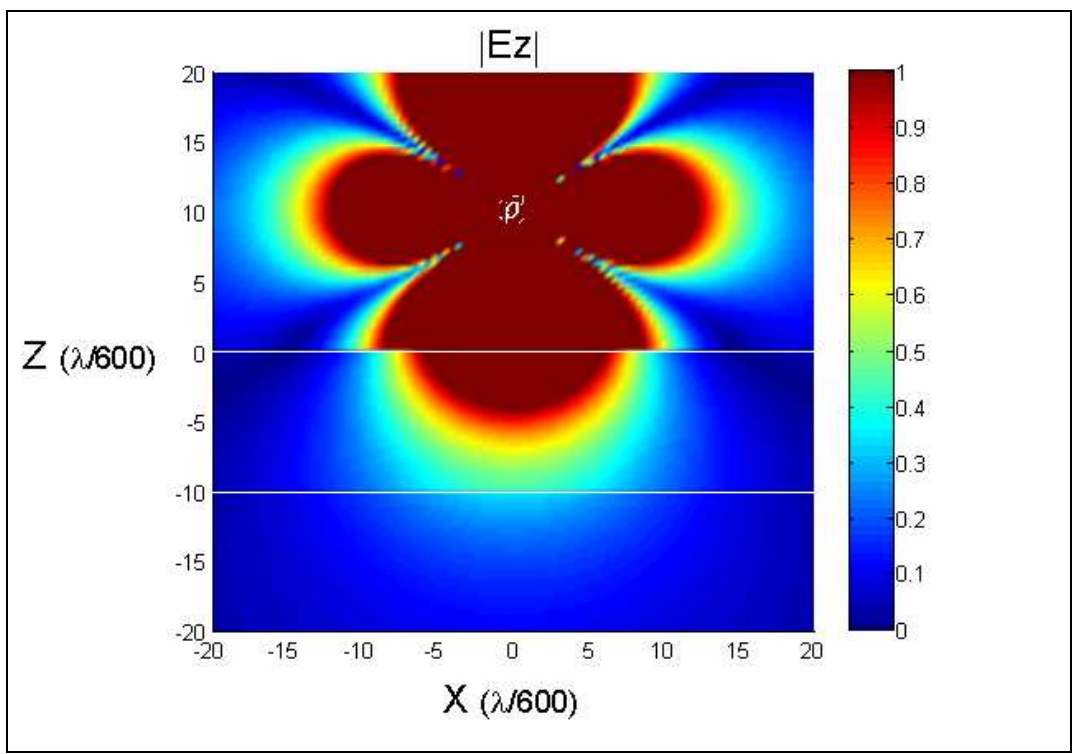

Figure 11: Sommerfeld calculation of the Ez field radiated by a Z-oriented dipole located in a medium 1 ( $\mu=1$ and $\varepsilon=2.25$ ), at a distance $\lambda / 60$ above a flat absorbing layer of thickness $\lambda / 60$ (medium $2, \mu=1$ and $\varepsilon=3+4 * \mathrm{i}$ ), and a substrate (medium $3, \mu=1$ and $\varepsilon=6.25$ ).

Figure 11 shows the SB-FSM computation of the problem.

For $\mathrm{Ns}=200$, we obtain a relative error of around $10^{-2}$ in an area of validity which is here also given by the length of the truncated interface. 


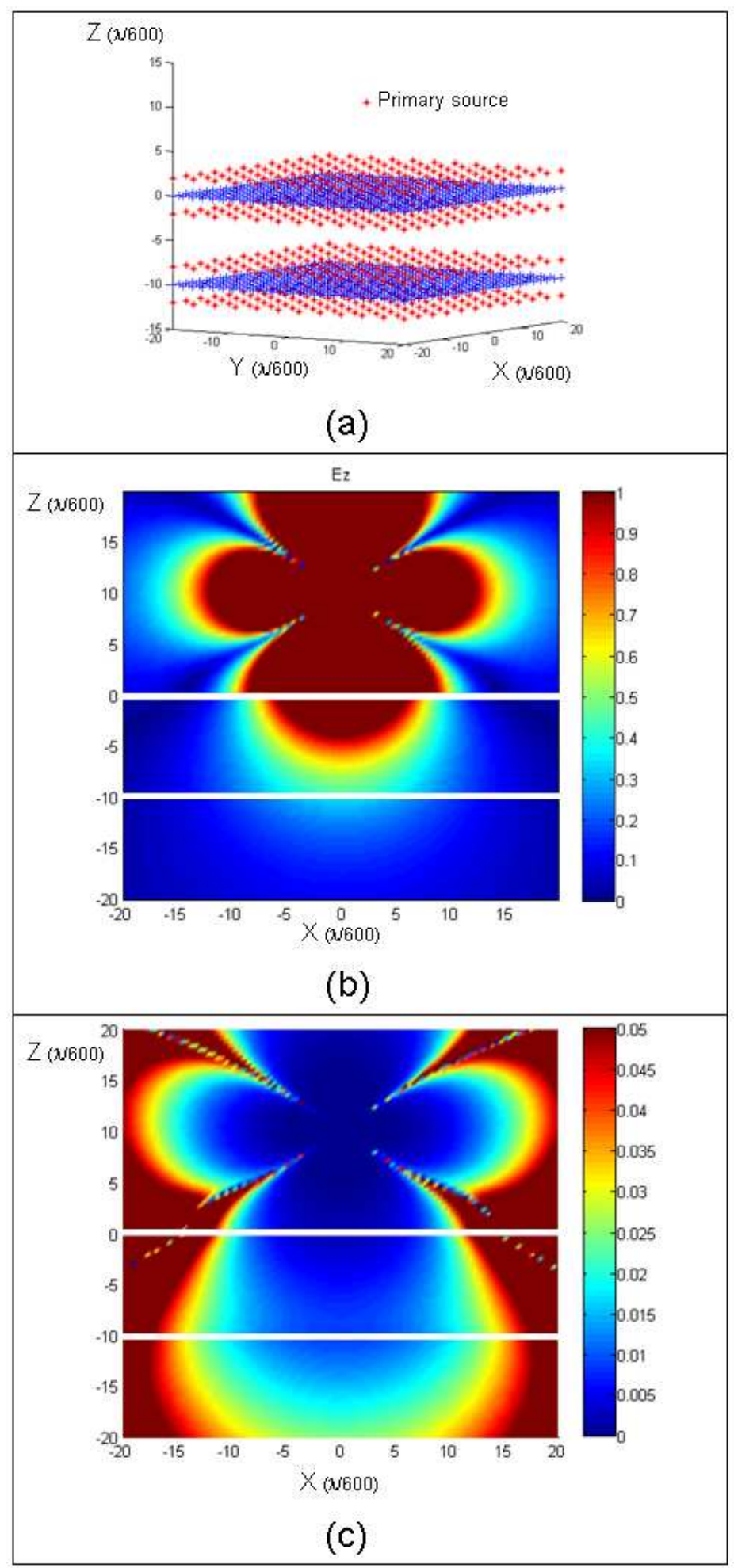

Figure 12: (a) SB-FSM setting of secondary sources (4 sets of 100 secondary sources, each constituted of two crossed fictitious dipoles tangential to the interfaces); (b) SB-FSM calculation of the dipole radiation in the three-media background; (c) Spatial relative error of SB-FSM calculation (fig. 12b) when compared to Sommerfeld calculation (Fig.11)

Computation times are equivalent for both methods in 3D. The reason is that Sommerfeld approach leads to a one-dimensional integration, as in 2D, whereas 3D SB-FSM requires 
much more unknowns and equations than 2D SB-FSM for similar accuracies. The SB-FSM can be however interesting in the case where the field must be computed over a very high number of spatial points (volumic integration for example), or, of course, for non-flat interfaces.

\section{Conclusion :}

We proposed an extension of the FSM for calculating the radiation of a 2D or 3D point-source in an open stratified background. This method has been shown to be faster than Sommerfeld method in $2 \mathrm{D}$ problems, less tricky to implement, and also adapted to non flat multilayers. It can be used to design multilayered emitting device (LEDs, ...), but also multilayer focusing devices, by the use of time-reversal techniques [27,28], for lithography or optical storage applications for example. We should also mention that SB-FSM is well suited to the optimization of such systems, because once the problem has been solved for a specific position of the point source, the computation for other source positions can be highly fast, due to the matricial formulation of the problem (the source position accounts only for the inhomogeneous part of the matricial equation of the problem).

In perspective, this method could be also very helpful when integrated into electromagnetic scattering techniques based on a decomposition of scatterer into small radiating pointsource(classical FSM, coupled dipole approximation, ...), for the computation of scattering by a target embedded in a flat or non-flat stratified background.

\section{Acknowledgements}

This work was supported by the French National Research Agency (ANR) through Carnot funding.

\section{References}

1. C. Hafner, The generalized Multipole Technique for Computational Electromagnetics (Artech House Books, Boston, 1990).

2. A. Sommerfeld, Ann. Phys. (Leipzig) 28, 665 (1909).

3. M. Paulus, P. Gay-Balmaz, and O.J.F. Martin, Phys. Rev. E 62, 5797 (2000)

4. W.C. Chew and S.Y. Chen, IEEE Ant. wir. Propag. Lett. 2, 254 (2003).

5. I.V. Lindell and E. Alanen, IEEE trans. Ant. Prop. 32, 1027 (1984),

6. L. Novotny, J. Opt. Soc. Am. A 14, 91 (1997)

7. D.G. Fang, J.J. Yang, G.Y. Delisle, IEE Proc. 135, 297 (1988)

8. M.A. Taubenblatt and T.K. Tran, J. Opt. Soc. Am. A 10, 912 (1993)

9. P. Gay-Balmaz and O.J.F martin, Opt. Comm. 184, 37 (2000) 
10. I.V. Lindell, A.H. Sihvola, K.O. Muinonen and P.W. Barbert, J. Opt. Soc. Am. A 8, 472 (1991)

11. M. Paulus, O.J.F. Martin, Phys. Rev. E 63, 066615 (2000)

12. B.R. Johnson, J. Opt. Soc. Am. A 9, 1341 (1992)

13. G. Tayeb, S. Enoch, J. Opt. Soc. Am. A 21, 1417 (2004)

14. R. Borghi, F. Gori, M. Santarsiero, F. Frezza and G. Schettini, J. Opt. Soc. Am. A13, 2441 (1996)

15. D. Kaklamani and H. T. Anastassiu, IEEE Ant. Propag. Mag. 44, 48 (2002).

16. G. Fikioris, IEEE Trans. Ant. Propag. 54, 2022 (2006).

17. F. Shubitidze, H. T. Anastassiuand, D. I. Kaklamani, IEEE Trans. Ant.and Propag. 52, 302 (2004).

18. P. J. Papakanellos, D. I. Kaklamani, and C. N. Capsalis, IEEE Trans. Ant. Propag. 49, $1491(2001)$.

19. M. Di Vico, F. Frezza, L. Pajewski and G. Schettini, IEEE Trans. Ant. Propag. 53, 719 (2005)

20. R. Borghi, F. Frezza, M. Santarsiero and G. Schettini, Int. Journ. of Inf. and Mill. Wav. 20, 1795 (1999)

21. R. H. MacPhie and K.L. Wu, IEEE Trans. Ant. Propag. 51, 2801 (2003).

22. P. Burghignoli and P.D. Cupis, Opt. Comm. 199, 17 (2001)

23. F. Frezza, L. Pajewski, D. Saccoccioni and G. Schettini, Opt. Comm. 265, 47 (2006)

24. A.H. Barnett, Journ. of Comput. Phys. 227, 7003 (2008)

25. P. Yeh, Optical Waves in Layered Media (Wiley, New York, 2005)

26.G. Benelli, Ph.D thesis, Université Paul Cézanne Aix-Marseille III, 2007.

27. A. Sentenac and P. Chaumet, PRL 101, 013901 (2008)

28. J.-J. Greffet and R. Carminati, Prog. Surf. Sci. 56, 133 (1997). 